\title{
Decay processes of a pseudoscalar $D(2900)$
}

\author{
Brenda B. Malabarba, ${ }^{1, *}$ K. P. Khemchandani, ${ }^{2, \dagger}$ and A. Martínez Torres ${ }^{1, \$}$ \\ ${ }^{1}$ Universidade de Sao Paulo, Instituto de Fisica, C.P. 05389-970, Sao Paulo, Brazil \\ ${ }^{2}$ Universidade Federal de São Paulo, C.P. 01302-907, São Paulo, Brazil
}

(Received 30 July 2021; accepted 31 October 2021; published 3 December 2021)

\begin{abstract}
We study the decay properties of a $D(2900)$ state, with spin parity $0^{-}$, whose existence was proposed in an earlier study of the $D K \bar{K}$ and coupled-channel system. It was found in the former work that a $D$ meson appears with a mass of about $2900 \mathrm{MeV}$ from the three-body dynamics while the charmless subsystem of the pseudoscalar mesons forms the $f_{0}(980)$ resonance. Motivated by the recent experimental investigations of $D$ mesons around $3000 \mathrm{MeV}$, we now study the two-body decays of $D(2900)$. We find that the nature of the said state sets the main decay channels to be $D^{*} \pi, D_{s}^{*} \bar{K}$ and $D_{s 0}^{*}(2317) \bar{K}$. It turns out that decay width to the last one is the largest, making the $D_{s 0}^{*}(2317) \bar{K}$ system to be the most favorable one to look for a signal of $D(2900)$. We compare the decay properties of our state with those of the $D$-meson states, proposed within quark models, near $3000 \mathrm{MeV}$. We hope that our findings and discussions can be useful for the future experimental investigations of charm mesons around $3000 \mathrm{MeV}$.
\end{abstract}

DOI: 10.1103/PhysRevD.104.116002

\section{INTRODUCTION}

With the access to the charm physics becoming more available in recent times, it seems possible to uncover the spectra of charm hadrons with certainty. In the last decade the BABAR and LHCb Collaborations have brought forward information on charm mesons in the mass region above $2.4 \mathrm{GeV}[1-4]$. Though the evidence for some of the $D / D^{*}$ states has been confirmed by both the Collaborations, the quantum numbers of such mesons are still under discussion. Structures at the highest mass known so far, around $3000 \mathrm{MeV}$, have been observed by the LHCb Collaboration $[2,3]$. In the former work, $\mathrm{LHCb}$ reports a signal around $3000 \mathrm{MeV}$ in the $D^{*+} \pi^{-}, D^{+} \pi^{-}$and $D^{0} \pi^{+}$ mass spectra. The structure found in the $D^{*+} \pi^{-}$spectrum is found to be compatible with an unnatural parity while that in the $D^{+} \pi^{-}$and $D^{0} \pi^{+}$spectra seems compatible with a natural parity assignment. The former one is denoted by $D_{J}(3000)$ and the latter by $D_{J}^{*}(3000)$ in Ref. [2]. Further, a spin $2 D$ meson has been found in the $D^{+} \pi^{-}$amplitude in a later work [3], and though it is labeled as $D_{2}^{*}(3000)$, the central value of its mass is $3200 \mathrm{MeV}$. Interestingly, in a more recent study of $B^{-}$decay to $D^{*+} \pi^{-} \pi^{-}$[4], no signal of

\footnotetext{
*brenda@if.usp.br

tkanchan.khemchandani@unifesp.br

*amartine@if.usp.br
}

Published by the American Physical Society under the terms of the Creative Commons Attribution 4.0 International license. Further distribution of this work must maintain attribution to the author(s) and the published article's title, journal citation, and DOI. Funded by SCOAP.
$D_{2}^{*}(3000)$ is found in the $D^{*} \pi$ system. All these findings have motivated a series of studies of the $D$-meson spectrum.

Different model calculations have been presented in Refs. [5-16] to understand the properties of $D_{J}(3000)$, within relativistic formalisms and by considering a variety of potentials, like an effective interaction arising from the sum of a one-gluon exchange term and long-range confining potentials, those based on heavy quark symmetry and chiral symmetry, etc. There seems to be a common finding in all these works, which is that states with quantum numbers $n^{2 s+1} J_{L}=2^{1} P_{1}, 2^{3} P_{1}, 3{ }^{1} S_{0}, 1^{1} F_{3}, 1^{3} F_{3}$ have a mass value of around $3000 \mathrm{MeV}$, and are all compatible with $D_{J}(3000)$. The decay properties of these aforementioned states, however, seem to be different. Authors of different works favor different spectroscopic assignments for $D_{J}(3000)$, though they cannot strongly exclude association with other possible quantum numbers since the information available from experiments is scarce and the quality of statistics of the data is poor at this point. For example, Refs. $[7,9,13,16]$ suggest attributing $2 P\left(1^{+}\right)$to $D_{J}(3000)$ and indicate systems like $D_{2}^{*}(2460) \pi, D^{*} \pi$, $D_{1}^{*}(2600) \pi$ to be important decay channels. On the other hand, the authors of Ref. [8] estimate the decay widths of the states related to $2^{1} P_{1}, 2^{3} P_{1}, 3^{1} S_{0}, 1^{1} F_{3}, 1^{3} F_{3}$ quantum numbers, including decays to lighter $D$ mesons in the spectra. In these latter calculations the widths of the $2 P$ and $1 F$ states turn out to be larger $(270-500 \mathrm{MeV})$ than in other works, which lay far from the experimental data. Thus, the authors exclude all possibilities except $3^{1} S_{0}$. On the basis of other arguments, Refs. $[10,11,15]$ suggest $3{ }^{1} S_{0}$ to be the favored quantum numbers and find significant branching ratios for the decay to $D \rho, D^{*} \rho, D^{*} \pi$, etc. 
The author of Ref. [17] proposes a very different description for $D_{J}$, which is that it can be either a $D f_{2}$ or $D_{1} \rho$ molecular state with $J^{\pi}=2^{-}$, though the two states (found in $D f_{2}$ and $D_{1} \rho$ systems) have very different widths. Besides, $D f_{2}$ and $D_{1} \rho$ could be treated as coupled channels.

From all these studies, one should expect a rich spectrum of $D$ mesons to show up in the $D^{*} \pi$ invariant mass distribution, around $3000 \mathrm{MeV}$. Though this does not seem to be the case so far, the picture should become clearer when higher statistics data are obtained in the future.

With the expectations of more experimental investigations occurring in the future, to better understand the properties of charmed mesons around $3000 \mathrm{MeV}$ and test the series of interesting predictions made by the works mentioned above, we find it timely to study the properties of a $J^{\pi}=0^{-} D$ meson whose existence was predicted by some of the authors of the present work in Ref. [18]. In this former work a $D$ meson with mass around $2900 \mathrm{MeV}$ was found to arise from the three-body dynamics in the $D K \bar{K}$ system. In Ref. [18], the same system was studied by solving few-body equations as well as through QCD sum rules by writing correlation functions in terms of currents representing the $D_{s 0}^{*}(2317) \bar{K}$ and $D f_{0}(980)$ systems. Both methods lead to the finding that a $D$-meson state, with spin parity $0^{-}$, arises with a mass around $2900 \mathrm{MeV}$. Further, a width of around $55 \mathrm{MeV}$ was determined from the threebody amplitude obtained in our former work. Incidentally, the formation of a state from $D f_{0}(980)$ dynamics was also concluded in Ref. [19], where a state with a mass around $2833 \mathrm{MeV}$ but with a narrower width was found. However, coupled channels like $D \pi \pi, D \pi \eta$ were not considered explicitly in Ref. [19], which can be the reason for finding a narrower width. We shall refer to this state as $D(2900)$ in the following discussions.

In the present work we study the main two-body decay channels of $D(2900)$ [18], which are $D^{*} \pi, D^{*} \bar{K}$ and $D_{s 0}^{*}(2317) \bar{K}$. We find that the decay width for $D(2900) \rightarrow$ $D_{s 0}^{*}(2317) \bar{K}$ turns out to be the largest and, thus, conclude that $D_{s 0}^{*}(2317) \bar{K}$ should be an ideal channel to look for a signal of $D(2900)$. We also discuss that the branching ratios of $D(2900)$ to decay channels considered as important for states predicted within quark models, like, $D^{*} \rho, D \rho$, $D_{2}^{*}(2460) \pi, D_{1}^{*}(2600) \pi$, etc., should be much smaller. Thus, $D(2900)$ can be distinguished from the states predicted by the quark models discussed above. Such findings should be useful in experimental studies of $D$ mesons around $3000 \mathrm{MeV}$.

\section{FORMALISM}

A $D$ meson arising from hadron coupled channel dynamics, studied within two distinct formalisms, was found in Ref. [18]. We find it useful to discuss the formalisms and findings of Ref. [18] briefly here, since the properties of the proposed $D(2900)$ are going to be essential in deducing its main decay mechanisms and decay channels. One of the formalisms considered in Ref. [18] consisted of solving few-body equations for the channels of three-pseudoscalar systems coupling to total charm +1 and strangeness zero: $D^{0} K^{+} K^{-}, D^{0} K^{0} \bar{K}^{0}, D^{0} \pi^{+} \pi^{-}, D^{0} \pi^{-} \pi^{+}$, $D^{0} \pi^{0} \pi^{0}, D^{0} \pi^{0} \eta, D^{+} K^{0} K^{-}, D^{+} \pi^{-} \pi^{0}, D^{+} \pi^{-} \eta, D^{+} \pi^{0} \pi^{-}$. The input two-body amplitudes were determined by solving the Bethe-Salpeter equation with the kernels deduced from chiral and heavy quark symmetry Lagrangians. Such two-body amplitudes carry the information of the dynamical generation of $D_{s}(2317)$ and scalar resonances: $\sigma(600), f_{0}(980)$ and $a_{0}(980)$, in the $D K, \pi \pi-K \bar{K}$ and $\pi \eta-K \bar{K}$ subsystems, respectively. That is, if the two-body amplitudes are scanned in an isospin configuration and energy region corresponding to the states mentioned above, they show formation of a resonance in the form of a peak on the real axis or in the form of a pole in the complex energy plane. The interaction in the remaining subsystem $D \bar{K}$ is also attractive. In fact, more recent investigations indicate formation of an exotic state in the $D \bar{K}$ system (see Ref. [20], which is an update of Ref. [21]). With such two-body amplitudes as inputs, we solve the three-body equations. To identify the nature of a possible state formed in the three-body system, one needs to project the amplitudes on an isospin basis. The three-body states can be labeled in terms of the total isospin and the isospin of a subsystem. Two configurations were studied in Ref. [18] for total isospin 1/2: (1) keeping the two charmless mesons in isospin 0 and (2) keeping the subsystem with positive strangeness and charm in isospin 0 . The three-body amplitude in the former configuration showed a distinct and dominant peak around $2900 \mathrm{MeV}$ when the invariant mass of the charmless subsystem was around the mass of $f_{0}(980)$. The findings of Ref. [18] were interpreted as formation of an effective $D f_{0}$ moleculelike state with mass around $2900 \mathrm{MeV}$.

The same problem was also studied within another formalism, based on QCD sum rules, in our previous work. In this case, two-point correlation functions were written in terms of interpolating molecular currents for the $D f_{0}$ and $D_{s}(2317) \bar{K}$ systems by attributing, for simplicity, $\bar{s} s$ and $\bar{s} c$ descriptions to $f_{0}(980)$ and $D_{s}(2317)$, respectively. A good convergence of the operator product expansion series was encountered by considering condensates up to dimension seven on the QCD side and by applying a Borel transformation. A pole plus continuum description was considered to describe the spectral density from the phenomenological point of view. As a consequence, stable mass values were found around $2900 \mathrm{MeV}$ in both cases, with the current-state coupling being two times bigger for the $D f_{0}$ current. The precise mass values obtained, with uncertainties, in the case of the $D f_{0}$ current can be summarized as $2926 \pm 237 \mathrm{MeV}$. It can be seen that, though simplified descriptions were considered for $f_{0}(980)$ and $D_{s}(2317)$, the results found were compatible with those obtained by solving few-body equations. 


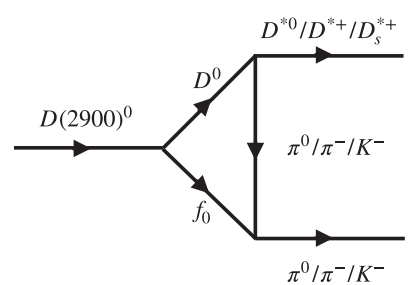

(a)

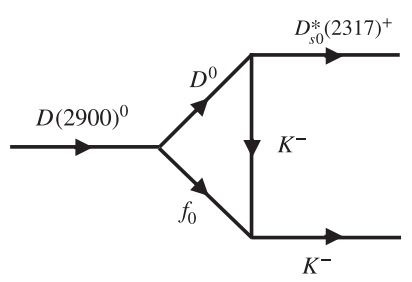

(b)
FIG. 1. Diagrams showing the decay of $D(2900)$ to the different possible final states.

Both studies indicate the existence of a $D$ meson with spin parity $0^{-}$and mass around $2900 \mathrm{MeV}$, arising, dominantly, from the $D f_{0}$ dynamics. A width of about $55 \mathrm{MeV}$ was determined for the state, from the three-body amplitude. Though such a mass value is compatible with that of $D_{J}(3000)$ discovered in the LHCb data [2], which is $2971.8 \pm 8.7 \mathrm{MeV}$, the width is smaller than the experimental value $188.1 \pm 44.8 \mathrm{MeV}$. In any case, it is difficult to discuss any relation between the two states since very limited information is available from the experimental data. The $D_{J}(3000)$ state in the experimental data appears close to the upper limit of the mass spectra, and, hence, systematic uncertainties on the properties of $D_{J}(3000)$ could not be determined in Ref. [2]. The results obtained in our present work should be useful in the identification of a $D$ meson with $D f_{0}$ molecular nature in future experimental investigations. Such a state would be a companion, although exotic in a different way, of the recently discovered $X(2900)$ states by the LHCb Collaboration [22].

We are now in a position to discuss the main decay channels of $D(2900)$. Since its nature is a $D f_{0}$ molecular state, it must primarily disintegrate into its constituents, which can subsequently interact, leading to other decay channels through a loop. Keeping in mind that the properties of $f_{0}(980)$ can be understood, essentially, by considering the contributions from $\bar{K} K$ and $\pi \pi$ dynamics [23], we can deduce the decay process of $D(2900)$ to proceed through the loops shown in Fig. 1. We can then enlist the main decay channels of the state with electric charge zero to be $D^{* 0} \pi^{0}, D^{*+} \pi^{-}, D_{s}^{*+} K^{-}$and $D_{s 0}^{*}(2317)^{+} K^{-}$.
We have already mentioned that $D(2900)$ and $f_{0}(980)$ can be interpreted as moleculelike states. We would like to add that similar is the case of $D_{s 0}^{*}(2317)$, which is interpreted as a $D K$ bound state within several model calculations [24-35], as well as from lattice QCD analyses [36-38]. In such a situation, the vertices $D(2900)^{0} \rightarrow$ $D^{0} f_{0}(980), D_{s 0}^{*}(2317)^{+} \rightarrow D^{0} K^{+}$and $f_{0} \rightarrow \pi^{0} \pi^{0}, \pi^{+} \pi^{-}$, $K^{+} K^{-}$, shown in Fig. 1, can be all written in terms of their respective couplings (summarized in Table I), together with the effective fields related to each of the mesons involved in the vertex. In Table I, we provide the couplings obtained from model calculations and compare them with those extracted from the experimental data or lattice simulations, when available. It can be seen that the values coming from the model calculations are in good agreement with the information known from the experimental/lattice data.

The coupling of the state $D(2900)^{0} \rightarrow D^{0} f_{0}(980)$, given in Table I, is calculated using the method followed in Refs. [41,42], where the two-body amplitude is assumed to be proportional to the three-body amplitude near the peak region. Following these former works, we can write $T_{D f_{0}}=\alpha T_{D[K \bar{K}]_{I=0}}$, where $\alpha$ is a proportionality constant, which can be determined using the unitarity condition for the $D f_{0}$ scattering amplitude:

$$
\mathcal{I} m\left\{T_{D f_{0}}^{-1}\right\}=\frac{\left|\vec{p}_{D f_{0}}\right|}{8 \pi \sqrt{S_{D f_{0}}}},
$$

with $\vec{p}_{D f_{0}}$ being the center of mass momentum and $\sqrt{s_{D f_{0}}}$ is taken as the mass of $D(2900)$. Using Eq. (1) and the three-body amplitude of Ref. [18], we can determine the relation between the effective $D f_{0}$ amplitude and $T_{D[K \bar{K}]_{I=0}}$. Further, assuming a Breit-Wigner form for the $D f_{0}$ amplitude, we can then determine the coupling $g_{D f_{0}}$ as

$$
g_{D f_{0}}^{2}=\alpha i M_{D(2900)} \Gamma_{D(2900)} T_{D[K \bar{K}]_{I=0}} .
$$

Using the value of the three-body amplitude, at the peak position, $T_{D[K \bar{K}]_{I=0}}$, we get $g_{D f_{0}}=(7259.63-i 667.579) \mathrm{MeV}$.

Considering now the value of $g_{D f_{0}}$, we can calculate the width of $D(2900)$ through

TABLE I. Couplings for the different vertices appearing in Fig. 1. We give the values obtained from model calculations as well as those determined from the available experimental data or lattice computations.

\begin{tabular}{lcc}
\hline \hline Vertex & Model couplings (MeV) & Experimental/lattice couplings \\
\hline$D(2900)^{0} \rightarrow D^{0} f_{0}(980)$ & $(7259.63-i 667.579)[18]$ & $\ldots$ \\
$f^{0} \rightarrow \pi^{0} \pi^{0}$ & $-\frac{1}{\sqrt{3}}(597.99-i 2028.5)[40]$ & $\frac{1}{\sqrt{2}}\left(-1430 \pm 10_{-60}^{+10+30}{ }_{-600}\right)[39]$ \\
$f_{0} \rightarrow \pi^{+} \pi^{-}$ & $-\frac{1}{\sqrt{3}}(597.99-i 2028.5)[40]$ & $-1430 \pm 10_{-60}^{+10+30}[39]$ \\
$f_{0} \rightarrow K^{+} K^{-}$ & $\frac{1}{\sqrt{2}}(3894.91+i 1328.01)[40]$ & $3760 \pm 40_{-80}^{+150+1160}[39]$ \\
$D_{s 0}^{*}(2317)^{+} \rightarrow D^{0} K^{+}$ & $-\frac{1}{\sqrt{2}}(9080 \pm 2530)[29]$ (in agreement with [28,30,31]) & $\frac{1}{\sqrt{2}}(12600 \pm 1500)[37]$ \\
\hline \hline
\end{tabular}




$$
\Gamma_{D(2900)}=\frac{1}{8 \pi} \frac{\left|\vec{p}_{D f_{0}}\right|}{M_{D(2900)}^{2}}\left|g_{D f_{0}}\right|^{2}
$$

and obtain a width of the order of $55 \mathrm{MeV}$, which indeed coincides with the value determined in Ref. [18].

To calculate the diagram in Fig. 1(a), we also require the following Lagrangian for the vector-pseudoscalar-pseudoscalar (VPP) vertex:

$$
\begin{aligned}
\mathcal{L}_{V P P} & =-i g_{V P P}\left\langle V_{\mu}\left[P, \partial_{\mu} P\right]\right\rangle \\
& =-i \mathcal{I}_{V P P} g_{V P P} V_{\mu}\left(\phi_{L} \partial^{\mu} \phi_{H}-\phi_{H} \partial^{\mu} \phi_{L}\right),
\end{aligned}
$$

where $\mathcal{I}_{V P P}$ is an isospin factor arising from the trace in the Lagrangian, $V_{\mu}$ is a (heavy) vector meson field, and $\phi^{H}$ $\left(\phi^{L}\right)$ represents heavy (light) pseudoscalar meson field. We use the following matrices for the mesons:

$$
\begin{aligned}
P & =\left(\begin{array}{cccc}
\frac{\pi^{0}}{\sqrt{2}}+\frac{\eta}{\sqrt{6}}+\frac{\eta_{c}}{\sqrt{12}} & \pi^{+} & K^{+} & \bar{D}^{0} \\
\pi^{-} & -\frac{\pi^{0}}{\sqrt{2}}+\frac{\eta}{\sqrt{6}}+\frac{\eta_{c}}{\sqrt{12}} & K^{0} & D^{-} \\
K^{-} & \bar{K}^{0} & \frac{-2 \eta}{\sqrt{6}}+\frac{\eta_{c}}{\sqrt{12}} & D_{s}^{-} \\
D^{0} & D^{+} & D_{s}^{+} & \frac{-3 \eta_{c}}{\sqrt{12}}
\end{array}\right), \\
V^{\mu} & =\left(\begin{array}{cccc}
\frac{\rho^{0}+\omega}{\sqrt{2}} & \rho^{+} & K^{*^{+}} & \bar{D}^{* 0} \\
\rho^{-} & \frac{-\rho^{0}+\omega}{\sqrt{2}} & K^{*^{0}} & D^{*-} \\
K^{*^{-}} & \bar{K}^{*^{0}} & \phi & D_{s}^{*-} \\
D^{* 0} & D^{*+} & D_{s}^{*+} & J / \psi
\end{array}\right) .
\end{aligned}
$$

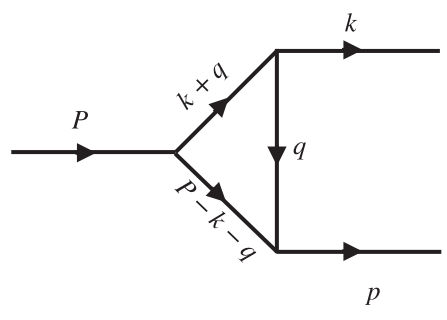

FIG. 2. Diagram showing momenta labels.

The coupling $g_{V P P}$ in Eq. (4) is determined as

$$
g_{V P P}=\frac{m_{\rho}}{2 f_{\pi}} \frac{m_{D^{*}}}{m_{K^{*}}} \sim 9.3
$$

where the factor $m_{D^{*}} / m_{K^{*}}$ has been included, following Ref. [43], to consider the presence of heavy mesons in the vertices.

Using the momenta label provided in Fig. 2, we can write Eq. (4) as

$$
\begin{aligned}
\mathcal{L}_{V P P} & =-i \mathcal{I}_{V P P} g_{V P P} \epsilon_{\mu}\left(-i[k+q]^{\mu}-i q^{\mu}\right) \\
& =-\mathcal{I}_{V P P} g_{V P P} \epsilon_{\mu}\left(k^{\mu}+2 q^{\mu}\right) .
\end{aligned}
$$

We can now write the amplitude for the diagram in Fig. 1(a), using the relation $i \mathcal{L}=-i t$, as

$$
\begin{aligned}
i t_{a} & =\int \frac{d^{4} q}{(2 \pi)^{4}} i t_{D(2900)^{0} \rightarrow D^{0} f_{0}} i t_{f^{0} \rightarrow P_{1} P_{2}} i t_{V P P} \frac{i}{(k+q)^{2}-m_{D}^{2}} \frac{i}{(P-k-q)^{2}-m_{f_{0}}^{2}} \frac{i}{q^{2}-m_{P_{1}}^{2}} \\
& =-\int \frac{d^{4} q}{(2 \pi)^{4}} \frac{g_{D(2900)^{0} \rightarrow D^{0} f_{0}} g_{f^{0} \rightarrow P_{1} P_{2}}\left[\mathcal{I}_{V P P} g_{V P P} \epsilon_{\mu}(k)\left(k^{\mu}+2 q^{\mu}\right)\right]}{\left[(k+q)^{2}-m_{D}^{2}\right]\left[(P-k-q)^{2}-m_{f_{0}}^{2}\right]\left[q^{2}-m_{P_{1}}^{2}\right]},
\end{aligned}
$$

where $m_{P_{1}}$ is the mass of the pseudoscalar meson with the momentum $q$. Using the Lorenz condition, the amplitude for the process becomes

$$
t_{a}=2 i g_{D(2900)^{0} \rightarrow D^{0} f_{0}} g_{f^{0} \rightarrow P_{1} P_{2}} \mathcal{I}_{V P P} g_{V P P} \epsilon_{\mu}(k)\left\{\int \frac{d^{4} q}{(2 \pi)^{4}} \frac{q^{\mu}}{\left[(k+q)^{2}-m_{D}^{2}\right]} \frac{1}{\left[(P-k-q)^{2}-m_{f_{0}}^{2}\right]\left[q^{2}-m_{P_{1}}^{2}\right]}\right\},
$$

with the values of $\mathcal{I}_{V P P}$ given in Table II. Further, following the Passarino-Veltman reduction for tensor integrals, we can write

$$
\begin{aligned}
t_{a}= & 2 i g_{D(2900)^{0} \rightarrow D^{0} f_{0}} g_{f^{0} \rightarrow P_{1} P_{2}} \mathcal{I}_{V P P} g_{V P P} \epsilon_{\mu}(k) \\
& \times\left\{a k^{\mu}+b P^{\mu}\right\},
\end{aligned}
$$

out of which only the second term survives, once again, due to the Lorenz condition. Hence, we do not need to find the
TABLE II. The values of the isospin factor, $\mathcal{I}_{V P P}$, obtained by calculating the trace in Eq. (4), for the different vectorpseudoscalar-pseudoscalar vertices shown in Fig. 1(a).

\begin{tabular}{lc}
\hline \hline Vertex & $\mathcal{I}_{V P P}$ \\
\hline$D^{0} D^{* 0} \pi^{0}$ & $-1 / \sqrt{2}$ \\
$D^{0} D^{*+} \pi^{-}$ & -1 \\
$D^{0} D_{s}^{*+} K^{-}$ & -1 \\
\hline \hline
\end{tabular}


coefficient $a$ but we need to determine $b$. For this, let us call the integral in Eq. (10) [which is equal to the terms in the curly bracket in Eq. (11)] $I^{\mu}$. Then, we can get a set of equations by contracting the integral with the different four-vectors:

$$
\begin{aligned}
& k \cdot I=a k^{2}+b k \cdot P \\
& P \cdot I=a P \cdot k+b P^{2},
\end{aligned}
$$

which leads to

$$
b=\frac{P \cdot k k \cdot I-k^{2} P \cdot I}{(k \cdot P)^{2}-k^{2} P^{2}},
$$

where

$$
\begin{aligned}
k \cdot I & =\int \frac{d^{4} q}{(2 \pi)^{4}} \frac{k \cdot q}{\left[(k+q)^{2}-m_{D}^{2}\right]\left[(P-k-q)^{2}-m_{f_{0}}^{2}\right]\left[q^{2}-m_{P_{1}}^{2}\right]}, \\
P \cdot I & =\int \frac{d^{4} q}{(2 \pi)^{4}} \frac{P \cdot q}{\left[(k+q)^{2}-m_{D}^{2}\right]\left[(P-k-q)^{2}-m_{f_{0}}^{2}\right]\left[q^{2}-m_{P_{1}}^{2}\right]} .
\end{aligned}
$$

Writing the previous equations explicitly in the center of mass frame, we have

$$
\begin{aligned}
k \cdot I & =\int \frac{d^{4} q}{(2 \pi)^{4}} \frac{k^{0} q^{0}-\vec{k} \cdot \vec{q}}{\left[(k+q)^{2}-m_{D}^{2}\right]\left[(P-k-q)^{2}-m_{f_{0}}^{2}\right]\left[q^{2}-m_{P_{1}}^{2}\right]}, \\
P \cdot I & =\int \frac{d^{4} q}{(2 \pi)^{4}} \frac{P^{0} q^{0}}{\left[(k+q)^{2}-m_{D}^{2}\right]\left[(P-k-q)^{2}-m_{f_{0}}^{2}\right]\left[q^{2}-m_{P_{1}}^{2}\right]} .
\end{aligned}
$$

To determine Eq. (13), we need to solve integrals on terms proportional to $\left(q^{0}\right)^{0}$ and to $\left(q^{0}\right)^{1}$. We can integrate Eqs. (15) and (16) on $q^{0}$ analytically, through Cauchy's theorem. To do this we rewrite Eqs. (15) and (16) to exhibit the $q^{0}$ dependence:

$$
\begin{aligned}
k \cdot I= & k^{0} \int \frac{d^{3} q}{(2 \pi)^{3}} \int \frac{d q^{0}}{(2 \pi)} \frac{q^{0}}{\left[\left(k^{0}+q^{0}\right)^{2}-\omega_{D}^{2}\right]\left[\left(P^{0}-k^{0}-q^{0}\right)^{2}-\omega_{f_{0}}^{2}\right]\left[\left(q^{0}\right)^{2}-\omega_{P_{1}}^{2}\right]} \\
& -\int \frac{d^{3} q}{(2 \pi)^{3}}(\vec{k} \cdot \vec{q}) \int \frac{d q^{0}}{(2 \pi)} \frac{1}{\left[\left(k^{0}+q^{0}\right)^{2}-\omega_{D}^{2}\right]\left[\left(P^{0}-k^{0}-q^{0}\right)^{2}-\omega_{f_{0}}^{2}\right]\left[\left(q^{0}\right)^{2}-\omega_{P_{1}}^{2}\right]}, \\
P \cdot I= & P^{0} \int \frac{d^{3} q}{(2 \pi)^{3}} \int \frac{d q^{0}}{(2 \pi)} \frac{q^{0}}{\left[\left(k^{0}+q^{0}\right)^{2}-\omega_{D}^{2}\right]\left[\left(P^{0}-k^{0}-q^{0}\right)^{2}-\omega_{f_{0}}^{2}\right]\left[\left(q^{0}\right)^{2}-\omega_{P_{1}}^{2}\right]},
\end{aligned}
$$

where

$$
\begin{aligned}
& \omega_{D}=\sqrt{(\vec{k}+\vec{q})^{2}+m_{D}^{2}}, \\
& \omega_{f_{0}}=\sqrt{(\vec{k}+\vec{q})^{2}+m_{f_{0}}^{2}}, \\
& \omega_{P_{1}}=\sqrt{\vec{q}^{2}+m_{P_{1}}^{2}} .
\end{aligned}
$$

Let us denote the integrand proportional to $\left(q^{0}\right)^{0}$ by $\mathcal{I}^{0}$ and the one proportional to $\left(q^{0}\right)^{1}$ by $\mathcal{I}^{1}$. Closing the contour clockwise in the complex plane, we get

$$
\int \frac{d q^{0}}{(2 \pi)} \mathcal{I}^{n}=-i \frac{\mathcal{N}_{n}}{\mathcal{D}}
$$

where 


$$
\begin{aligned}
\mathcal{N}_{0}= & 2 P^{0} k^{0} \omega_{f_{0}} \omega_{P_{1}}-\left(P^{0}\right)^{2} \omega_{f_{0}}\left(\omega_{P_{1}}+\omega_{D}\right)+\left(\omega_{f_{0}}+\omega_{D}\right)\left[\left(\omega_{f_{0}}+\omega_{P_{1}}\right)\left(\omega_{P_{1}}+\omega_{D}\right)\right. \\
& \left.\times\left(\omega_{f_{0}}+\omega_{D}+\omega_{P_{1}}\right)-\omega_{P_{1}}\left(k^{0}\right)^{2}\right], \\
\mathcal{N}_{1}= & \omega_{P_{1}}\left[P^{0} \omega_{D}\left(\omega_{D}+\omega_{P_{1}}\right)\left(2 \omega_{f_{0}}+\omega_{D}+\omega_{P_{1}}\right)+\left(P^{0}\right)^{2} \omega_{f_{0}} k^{0}-P^{0}\left(2 \omega_{f_{0}}+\omega_{D}\right)\left(k^{0}\right)^{2}\right. \\
& \left.-\left(\omega_{f_{0}}+\omega_{D}\right) k^{0}\left(\omega_{f_{0}}^{2}+\left(\omega_{P_{1}}+\omega_{D}\right)^{2}+\omega_{f_{0}}\left(2 \omega_{P_{1}}+\omega_{D}\right)-\left(k^{0}\right)^{2}\right)\right],
\end{aligned}
$$

and

$$
\begin{aligned}
\mathcal{D}= & 2 \omega_{P_{1}} \omega_{f_{0}} \omega_{D}\left(P^{0}-\omega_{f_{0}}-\omega_{D}+i \epsilon\right)\left(-P^{0}-\omega_{f_{0}}-\omega_{P_{1}}+k^{0}+i \epsilon\right)\left(-\omega_{P_{1}}-\omega_{D}+k^{0}+i \epsilon\right) \\
& \times\left(P^{0}-\omega_{f_{0}}-\omega_{P_{1}}-k^{0}+i \epsilon\right)\left(\omega_{P_{1}}+\omega_{D}+k^{0}\right)\left(P^{0}+\omega_{f_{0}}+\omega_{D}\right) .
\end{aligned}
$$

Eventually, in the calculations, we replace $-\omega_{f_{0}}+i \epsilon \rightarrow-\omega_{f_{0}}+i \Gamma_{f_{0}} / 2$ in Eq. (22), to take into account the unstable nature of $f_{0}$.

To summarize, we calculate the amplitude in Fig. 1(a) as

$$
\begin{aligned}
t_{a}= & 2 i g_{D(2900)^{0} \rightarrow D^{0} f_{0}} g_{f^{0} \rightarrow P_{1} P_{2}} \mathcal{I}_{V P P} g_{V P P} \frac{\epsilon_{\mu}(k) P^{\mu}}{\left(k^{0} P^{0}\right)^{2}-k^{2} P^{2}}\left\{P ^ { 0 } k ^ { 0 } \left[k^{0} \int \frac{d^{3} q}{(2 \pi)^{3}}\left(-i \frac{\mathcal{N}_{1}}{\mathcal{D}}\right)\right.\right. \\
& \left.\left.-\int \frac{d^{3} q}{(2 \pi)^{3}}(\vec{k} \cdot \vec{q})\left(-i \frac{\mathcal{N}_{0}}{\mathcal{D}}\right)\right]-k^{2} P^{0} \int \frac{d^{3} q}{(2 \pi)^{3}}\left(-i \frac{\mathcal{N}_{1}}{\mathcal{D}}\right)\right\} .
\end{aligned}
$$

The amplitude for the decay process shown in Fig. 1(b) can be written by replacing the VPP vertex by the coupling $g_{D_{s 0}^{*}(2317) \rightarrow D K}$ in Eq. (9), finding

$$
\begin{aligned}
t_{b}= & i g_{D(2900)^{0} \rightarrow D^{0} f_{0}} g_{f^{0} \rightarrow P_{1} P_{2}} g_{D_{s 0}^{*}(2317) \rightarrow D K}\left\{\int \frac{d^{4} q}{(2 \pi)^{4}} \frac{1}{\left[(k+q)^{2}-m_{D}^{2}\right]}\right. \\
& \left.\times \frac{1}{\left[(P-k-q)^{2}-m_{f_{0}}^{2}\right]\left[q^{2}-m_{P_{1}}^{2}\right]}\right\} \\
= & i g_{D(2900)^{0} \rightarrow D^{0} f_{0}} g_{f^{0} \rightarrow P_{1} P_{2}} g_{D_{s 0}^{*}(2317) \rightarrow D K} \int \frac{d^{3} q}{(2 \pi)^{3}}\left(-i \frac{\mathcal{N}_{0}}{\mathcal{D}}\right) .
\end{aligned}
$$

It should be mentioned that the three-momentum integrals appearing in Eqs. (23) and (24) are convergent.

\section{RESULTS AND DISCUSSION}

Having calculated the amplitudes, we can determine the partial decay widths of $D(2900)$ using Eq. (3). Before showing the results, we must discuss the uncertainties present in the formalism. Among the couplings given in Table I, besides taking the uncertainty on the value for $D_{s 0}^{*}(2317)^{+} \rightarrow D^{0} K^{+}$from Ref. [29], we consider a $10 \%$ error on the other couplings too. Such an error on the $D(2900)^{0} \rightarrow D^{0} f_{0}(980)$ coupling is consistent with varying the width of $D(2900)$ in $55 \pm 10 \mathrm{MeV}$. Additionally, we take the mass for $D(2900)$ in the range $2900 \pm 50 \mathrm{MeV}$ and for $f_{0}(980)$ as $990 \pm 20 \mathrm{MeV}$ [23]. To take into account all the uncertainties, random numbers are generated within the range of all the inputs and mean values as well as standard deviations on the results are evaluated.

The results obtained are given in Table III. It can be seen that the decay width to a $D_{s 0}^{*}(2317)^{+} K^{-}$final state is the largest of all; it turns out to be about 40-100 times bigger than the widths to the other channels. Such findings imply that $D_{s 0}^{*}(2317)^{+} K^{-}$, rather than $D^{*} \pi$ analyzed in Ref. [2], should be a far more promising channel to look for a signal of $D(2900)$ which is a $D f_{0}(980)$ moleculelike state.

We would now like to discuss that the mechanisms of decay of $D(2900)$ to final states like $D^{*} \rho, D \rho$, $D_{2}^{*}(2460) \pi, D_{1}^{*}(2600) \pi$ involve higher-order loops, due to the $D f_{0}(980)$ molecular nature of $D(2900)$. We show

TABLE III. Partial widths of $D(2900)$ to the main two-body decay channels.

\begin{tabular}{lc}
\hline \hline Decay channel & Decay width $(\mathrm{MeV})$ \\
\hline$D^{* 0} \pi^{0}$ & $0.18 \pm 0.04$ \\
$D^{*+} \pi^{-}$ & $0.35 \pm 0.07$ \\
$D_{s}^{*+} K^{-}$ & $0.44 \pm 0.10$ \\
$D_{s 0}^{*}(2317)^{+} K^{-}$ & $18.33 \pm 7.25$ \\
\hline \hline
\end{tabular}




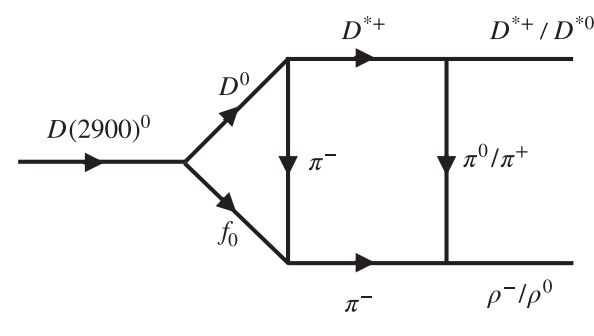

(a)

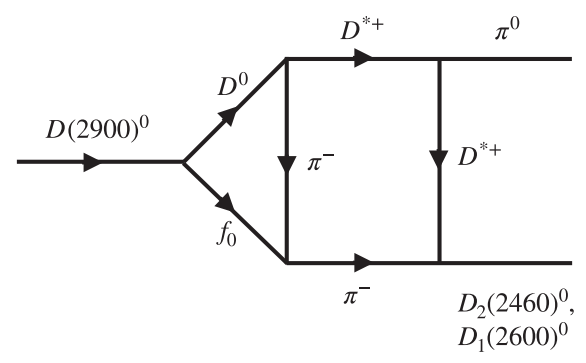

(b)

FIG. 3. Decay mechanism of $D(2900)^{0}$ to final states like $D^{*+} \rho^{-}, D^{*+} \rho^{-}, D_{2}^{* 0}(2460) \pi^{0}$ and $D^{* 0}(2600) \pi^{0}$.

some examples in Fig. 3 of the decay processes to the mentioned final states. Similar will be the mechanisms to yet other channels, like $D^{*} \omega, D \omega$. Such mechanisms imply suppressed partial widths to such channels. Thus, our state can be distinguished from the states predicted within the quark model calculations [5-16]. We hope that our present study can be useful in investigation of charm meson in the region around $3000 \mathrm{MeV}$.

\section{ACKNOWLEDGMENTS}

B. B. M., K.P. K. and A. M. T. gratefully acknowledge the support from the Fundação de Amparo à Pesquisa do Estado de São Paulo (FAPESP), Processos n ${ }^{\circ}$ 2020/006768, 2019/17149-3 and 2019/16924-3, respectively. K. P. K. and A. M. T. are also thankful to the Conselho Nacional de Desenvolvimento Científico e Tecnológico (CNPq) for Grants No. 305526/2019-7 and No. 303945/2019-2.
[1] P. del Amo Sanchez et al. (BABAR Collaboration), Phys. Rev. D 82, 111101 (2010).

[2] R. Aaij et al. (LHCb Collaboration), J. High Energy Phys. 09 (2013) 145.

[3] R. Aaij et al. (LHCb Collaboration), Phys. Rev. D 94, 072001 (2016).

[4] R. Aaij et al. (LHCb Collaboration), Phys. Rev. D 101, 032005 (2020).

[5] D. Ebert, R. N. Faustov, and V. O. Galkin, Eur. Phys. J. C 66, 197 (2010).

[6] Y. Sun, X. Liu, and T. Matsuki, Phys. Rev. D 88, 094020 (2013).

[7] G. L. Yu, Z. G. Wang, Z. Y. Li, and G. Q. Meng, Chin. Phys. C 39, 063101 (2015).

[8] Q. F. L and D. M. Li, Phys. Rev. D 90, 054024 (2014).

[9] L. Y. Xiao and X. H. Zhong, Phys. Rev. D 90, 074029 (2014).

[10] S. Godfrey and K. Moats, Phys. Rev. D 93, 034035 (2016).

[11] Q. T. Song, D. Y. Chen, X. Liu, and T. Matsuki, Phys. Rev. D 92, 074011 (2015).

[12] M. Batra and A. Upadhayay, Eur. Phys. J. C 75, 319 (2015).

[13] S. C. Li, T. Wang, Y. Jiang, X. Tan, Q. Li, G. L. Wang, and C. H. Chang, Phys. Rev. D 97, 054002 (2018).

[14] P. Gupta and A. Upadhyay, Phys. Rev. D 97, 014015 (2018).

[15] A. M. Badalian and B. L. G. Bakker, Phys. At. Nucl. 84, 354 (2021).

[16] K. Gandhi and A. K. Rai, Eur. Phys. J. A 57, 23 (2021).

[17] C. W. Xiao, Eur. Phys. J. A 53, 176 (2017).

[18] A. Martinez Torres, K. P. Khemchandani, M. Nielsen, and F. S. Navarra, Phys. Rev. D 87, 034025 (2013).
[19] V. R. Debastiani, J. M. Dias, and E. Oset, Phys. Rev. D 96, 016014 (2017).

[20] R. Molina and E. Oset, Phys. Lett. B 811, 135870 (2020).

[21] R. Molina, T. Branz, and E. Oset, Phys. Rev. D 82, 014010 (2010).

[22] R. Aaij et al. (LHCb Collaboration), Phys. Rev. D 102, 112003 (2020).

[23] P. A. Zyla et al. (Particle Data Group), Prog. Theor. Exp. Phys. 2020, 083C01 (2020).

[24] E. van Beveren and G. Rupp, Phys. Rev. Lett. 91, 012003 (2003).

[25] T. Barnes, F. E. Close, and H. J. Lipkin, Phys. Rev. D 68, 054006 (2003).

[26] E. E. Kolomeitsev and M. F. M. Lutz, Phys. Lett. B 582, 39 (2004).

[27] A. P. Szczepaniak, Phys. Lett. B 567, 23 (2003).

[28] T. Mehen and R. P. Springer, Phys. Rev. D 72, 034006 (2005).

[29] D. Gamermann, E. Oset, D. Strottman, and M. J. Vicente Vacas, Phys. Rev. D 76, 074016 (2007).

[30] F. K. Guo, P. N. Shen, H. C. Chiang, R. G. Ping, and B. S. Zou, Phys. Lett. B 641, 278 (2006).

[31] A. Faessler, T. Gutsche, V. E. Lyubovitskij, and Y. L. Ma, Phys. Rev. D 76, 014005 (2007).

[32] J. M. Flynn and J. Nieves, Phys. Rev. D 75, 074024 (2007).

[33] L. Liu, K. Orginos, F. K. Guo, C. Hanhart, and U. G. Meissner, Phys. Rev. D 87, 014508 (2013).

[34] M. Cleven, H. W. Griehammer, F. K. Guo, C. Hanhart, and U. G. Meiner, Eur. Phys. J. A 50, 149 (2014).

[35] M. Albaladejo, D. Jido, J. Nieves, and E. Oset, Eur. Phys. J. C 76, 300 (2016). 
[36] D. Mohler, C. B. Lang, L. Leskovec, S. Prelovsek, and R. M. Woloshyn, Phys. Rev. Lett. 111, 222001 (2013).

[37] A. Martínez Torres, E. Oset, S. Prelovsek, and A. Ramos, J. High Energy Phys. 05 (2015) 153.

[38] G. K. C. Cheung, C. E. Thomas, D. J. Wilson, G. Moir, M. Peardon, and S. M. Ryan (Hadron Spectrum Collaboration), J. High Energy Phys. 02 (2021) 100.

[39] F. Ambrosino et al. (KLOE Collaboration), Eur. Phys. J. C 49, 473 (2007).
[40] J. A. Oller and E. Oset, Nucl. Phys. A620, 438 (1997); A652, 407(E) (1999).

[41] A. Martinez Torres, K. P. Khemchandani, L. S. Geng, M. Napsuciale, and E. Oset, Phys. Rev. D 78, 074031 (2008).

[42] B. B. Malabarba, X. L. Ren, K. P. Khemchandani, and A. Martinez Torres, Phys. Rev. D 103, 016018 (2021).

[43] W. H. Liang, C. W. Xiao, and E. Oset, Phys. Rev. D 89, 054023 (2014). 